\title{
A method for utilizing a single port for multiple functions on the IBM PC or compatible system
}

\author{
KARL R. ZURN \\ MED Associates, Inc., East Fairfield, Vermont \\ THOMAS A. TATHAM \\ Temple University School of Medicine, Philadelphia, Pennsylvania \\ and \\ TOM REPSTAD \\ MED Associates, Inc, East Fairfield, Vermont
}

\begin{abstract}
Psychological experiments often require the control of a large number of output lines; unfortunately, the IBM PC provides only 32 8-bit ports through which communication may be implemented without risk of conflict with other software/hardware. A single 8-bit port may be used to control a large number of devices by adding appropriate decoder logic between the computer's port and the devices that are to be controlled. Software and hardware are presented that illustrate this technique by controlling an 8-digit light-emitting diode display with a single IBM PC output port.
\end{abstract}

Many psychological preparations involve sending and receiving large numbers of electrical signals to and from an experimental apparatus. For example, a single IBM PC may control dozens of chambers with the use of photobeam detectors to monitor the activity levels of a large number of rodents. Similarly, IEE stimulus projectors used in discrimination research with pigeons contain 12 independently activated elements; furthermore, each experimental chamber may contain several projectors and the computer may simultaneously control multiple operant chambers. Although there are 1,024 8-bit input/output (I/O) ports assigned on the PC, and one device may be controlled by each bit, there are only 32 absolutely safe addresses (768-799 decimal) that will not conflict with other functions. Some interface systems, such as the MED Associates, Inc. interface (Tatham \& Zurn, 1989), specifically address only 16 locations (780-795 decimal) for $\mathrm{I} / \mathrm{O}$ control functions. It is clear that a method is needed to functionally extend the number of available ports.

Conventional output-interfacing schemes involve a single-stage process in which a single 8-bit byte is passed to one of the 32 safe hardware ports. Similar techniques for the Commodore 64 were discussed by Wynne (1990). The interface circuitry then uses the bit pattern contained in the byte to determine the appropriate status of a set of 8 outputs. For example, to turn on outputs 1 and 2 of an

The authors acknowledge Jack J. McDowell and Mark Pevey of Emory University for testing the LED display unit. Thomas A. Tatham is now at the Department of Psychiatry, Uniform Services University of the Health Sciences, Bethesda, MD. Requests for reprints may be sent to Karl R. Zum, MED Associates, Inc., Box 47, East Fairfield, VT 05448. operant chamber attached to port 780 , one would pass a value of 3 (00000011 binary) to port 780 . Unfortunately, this scheme limits one to controlling 128 devices.

We were hampered by these limitations in a project in which we needed to control an eight-digit light-emitting diode (LED) display. The display was designed for use in human experiments to provide subjects with online feedback about the number of points earned during experimental sessions. The display unit consists of eight adjacent LED units, each providing for a digit and a decimal point. Each of the eight digits would require nine control lines (eight to form the digits 0 through 9 , blank and minus, and one to control the decimal point). If a simple software-driving scheme were used without additional front-end hardware, nine ports (one for each of the eight digits and one for the eight decimal points) would be needed. Although a PC provides enough output ports to drive one LED display, we wanted to be able to simultaneously control several LED displays. By adopting a "multiplexing" scheme, we were able to overcome port limitations with techniques that should be useful in a wide variety of applications to control a far larger number of devices than is normally possible with a PC. Conceptually, a port may be multiplexed by connecting it to address decoder circuitry that is then used to route data passed through the port to its target location. Specifically, one could pass an address byte through the port to the address decoder, followed by a data byte containing a bit pattern that is used to actually control the experimental apparatus. With this 2-byte scheme, a single IBM port may be used to control 256 8-bit addresses (yielding 2,048 control lines per port). 


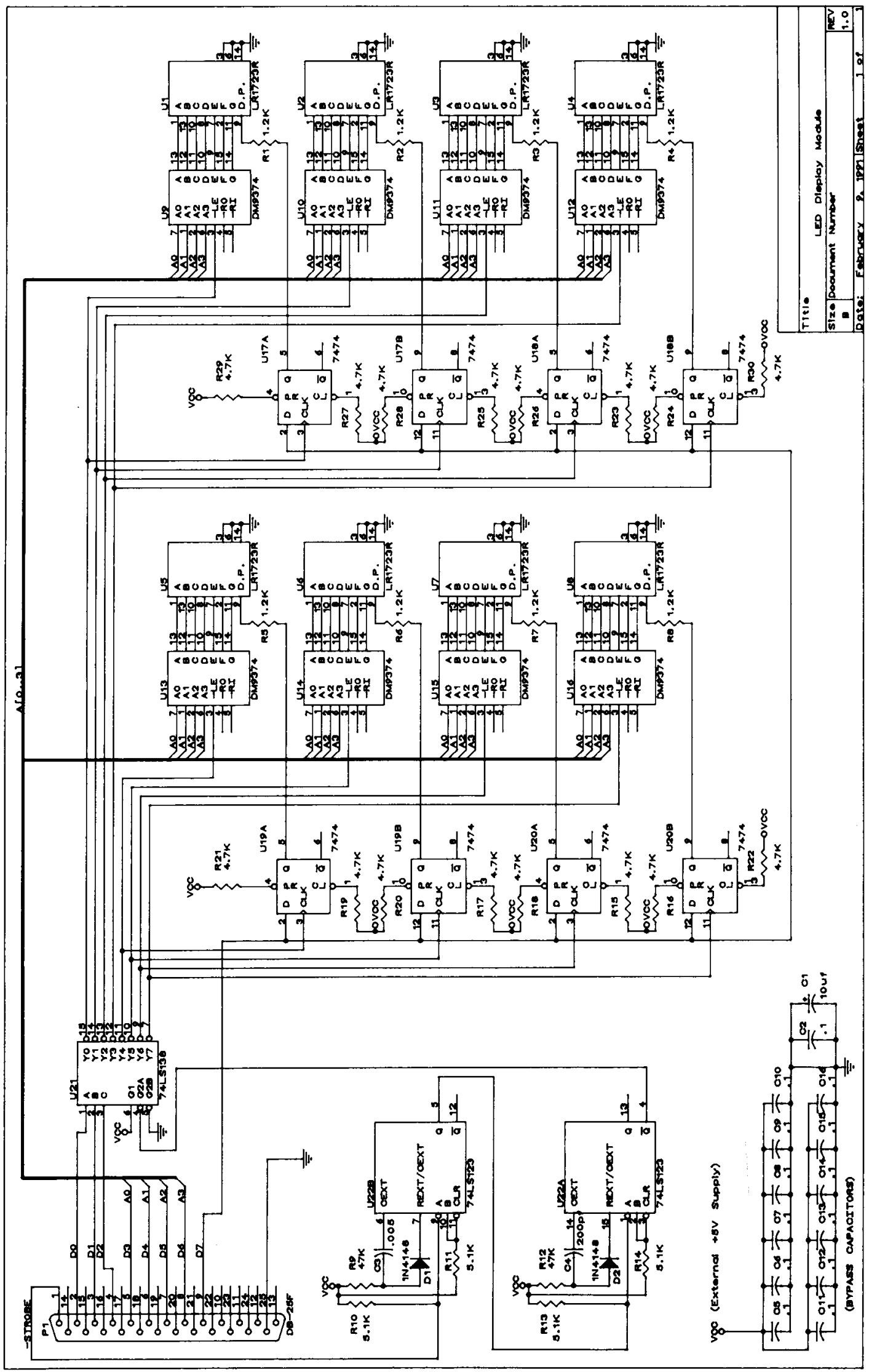


A more limited approach to multiplexing is to combine address and control data into a single byte. We selected this technique as the basis for the hardware and software system we developed for controlling the LED display. Each byte is processed by custom front-end hardware that was developed for this project (see the schematic in Figure 1) and that uses the information in the byte to select a given LED and then display a digit and/or a decimal point. Bits 0-2 of the byte serve as address bits to select a particular LED within the eight-LED array, bits 3-6 encode the numeric value of the digit to be displayed (expressed as an extended binary coded decimal, or EBCD), and bit 7 contains the off/off status of the decimal point. Table 1 provides a complete listing of the layout of the control byte and the addresses and EBCD values from which the byte is constructed.

\section{HARDWARE}

The hardware that processes the hybrid address/data bytes sent from the PC consists of a MED Associates DIG700 16-port interface, SG-6000C cabinet and power supply, and a DIG-720A single-port (8-bit) output module. The MED Associates interface system has the advantage of requiring only a single instruction to write data to the port, but almost any transistor-to-transistor logic (TTL) level port adapter compatible with the architecture of the IBM PC could be used. For instance, the display driver could be connected to a standard IBM PC parallel port (IBM Corporation, 1984); the pin-out connections of the DB-25F connector (P1) can be plugged directly into the parallel port.

Figure 1 shows the display unit and the circuitry developed to control it. The operation is as follows: Data bits 0-2 are input to U21 (a 74LS138 3-line to 8-line decoder). The eight outputs from U21 are used to enable U9-U16 (DM9374) memory-equipped display drivers, as well as the corresponding decimal-point memory U17-U20
(7474). Data bits 3-6 contain the binary-coded decimal data to be written to the display drivers U9-U16 (DM9374). Data bit 7 contains decimal-point information. Output D8, delayed by one-shot U22 (74LS123), is the 1/O write pulse used to enable U21 (74LS138), whose outputs will be coincident with the pulse. The write pulse is delayed so that the data will be stable prior to its transfer to a specific LED. Table 2 shows the parts list for the display unit.

\section{SOFTWARE}

Writing software to control the display circuitry is straightforward. Complete numbers are displayed by outputting a sequence of bytes, with each byte representing the address and value of successive digits in the display. For example, using the data contained in Table 1, the number 2.1 could be output via port 780 (the address location selected for the DIG-720A) by issuing a pair of bytes. The first byte, used to set the second LED in the display (numbered from right to left) to 2 ., would be set to binary 10010001 (decimal 145). Bits 0-2 are set to 001 to select the second LED, bits 3-6 are set to 0010 , because this is the EBCD representation of 2, and bit 7 is set to 1 to indicate that the decimal point is turned on. To complete the display of 2.1 , a second byte set to binary 00001000 (decimal 8) would be output to select the first LED (address 000), set the digit display to 1 (EBCD 0001 ), and disable the decimal point (bit 7 is set to 0 ).

Displaying a number may be further simplified by writing subroutines to generate and send the appropriate sequence of bytes. Display-driver software that maps a floating-point number into 8 bytes of data to control the display is shown in the Appendix.

The display-driver subroutines are written in Turbo Pascal and may be easily called by any Turbo Pascal program, including MED-PC (Tatham \& Zurn, 1989). The display procedures (a procedure is the Pascal nomencla-

Table 1

Layout of Byte Used to Control LED Display

\begin{tabular}{|c|c|c|c|c|c|}
\hline \multicolumn{2}{|c|}{$\begin{array}{c}\text { Bit } 7 \\
\text { (Decimal Point Status) }\end{array}$} & \multicolumn{2}{|c|}{$\begin{array}{c}\text { Bits 6-3 } \\
\text { (EBCD of Character to Display) }\end{array}$} & \multicolumn{2}{|c|}{$\begin{array}{c}\text { Bits 2-0 } \\
\text { (Selects LED to Manipulate) }\end{array}$} \\
\hline $\begin{array}{l}\text { D.P. } \\
\text { Status }\end{array}$ & $\begin{array}{c}\text { Bit } \\
\text { Value }\end{array}$ & $\begin{array}{c}\text { Character } \\
\text { to Display }\end{array}$ & $\begin{array}{l}\text { EBCD } \\
\text { Code }\end{array}$ & LED \# & $\begin{array}{l}\text { LED } \\
\text { Address }\end{array}$ \\
\hline $\begin{array}{l}\text { ON } \\
\text { OFF }\end{array}$ & $\begin{array}{l}0 \\
1\end{array}$ & $\begin{array}{c}0 \\
1 \\
2 \\
3 \\
4 \\
5 \\
6 \\
7 \\
8 \\
9 \\
- \\
\text { Blank }\end{array}$ & $\begin{array}{l}0000 \\
0001 \\
0010 \\
0011 \\
0100 \\
0101 \\
0110 \\
0111 \\
1000 \\
1001 \\
1010 \\
1111\end{array}$ & $\begin{array}{l}1 \\
2 \\
3 \\
4 \\
5 \\
6 \\
7 \\
8\end{array}$ & $\begin{array}{l}000 \\
001 \\
010 \\
011 \\
100 \\
101 \\
110 \\
111\end{array}$ \\
\hline
\end{tabular}


Table 2

Parts List for 8-Digit LED Display

\begin{tabular}{|c|c|c|}
\hline Quantity & Type & Ref Deisgnators \\
\hline \multicolumn{3}{|c|}{ Capacitors } \\
\hline 1 & $10 \mu \mathrm{F} 10 \mathrm{~V}$ Electrolytic & $\mathrm{Cl}$ \\
\hline 14 & $.1 \mu \mathrm{F} 50 \mathrm{~V}$ & $\begin{array}{l}\mathrm{C} 2, \mathrm{C} 5, \mathrm{C} 6, \mathrm{C} 7, \mathrm{C} 8, \mathrm{C} 9, \mathrm{C} 10, \mathrm{C} 11, \\
\mathrm{C} 12, \mathrm{C} 13, \mathrm{C} 14, \mathrm{C} 15, \mathrm{C} 16, \mathrm{C} 17, \mathrm{C} 18\end{array}$ \\
\hline 1 & $.005 \mu \mathrm{F} 50 \mathrm{~V}$ & $\mathrm{C} 3$ \\
\hline 1 & $200 \mathrm{pF} 50 \mathrm{~V}$ & $\mathrm{C} 4$ \\
\hline \multicolumn{3}{|c|}{ Diodes } \\
\hline 2 & $1 \mathrm{~N} 4148$ & D1,D2 \\
\hline \multicolumn{3}{|c|}{ Resistors } \\
\hline 8 & $220 \Omega$ & R1,R2,R3,R4,R5,R6,R7,R8 \\
\hline 2 & $47 \mathrm{~K}$ & R9,R12 \\
\hline 4 & $5.1 \mathrm{~K}$ & $\mathbf{R} 10, \mathbf{R} 11, \mathbf{R} 13, \mathbf{R} 14$ \\
\hline \multicolumn{3}{|c|}{ Integrated Circuits } \\
\hline 4 & 7474 & $\mathrm{U} 17, \mathrm{U} 18, \mathrm{U} 19, \mathrm{U} 20$ \\
\hline 1 & $74 \mathrm{LS} 123$ & $\mathrm{U} 22$ \\
\hline 1 & $74 \mathrm{LS} 138$ & $\mathrm{U} 21$ \\
\hline 8 & DM9374* & U9,U10,U11,U12,U13,U14,U15,U16 \\
\hline \multicolumn{3}{|c|}{ LED Displays } \\
\hline 8 & LR1723R† & U1,U2,U3,U4,U5,U6,U7,U8 \\
\hline
\end{tabular}

ture for a subroutine) are nested within a unit (a unit is a Turbo Pascal linkable object module) named DISP, in which there are two global procedures: DISPLAY places a floating-point number on the LED display, whereas Disp__off blanks the display.

To display a floating-point number, one calls DISPLAY with three arguments: the first is the value of the number to be displayed, the second is the number of digits to follow the decimal point, and the third is the port address of the display hardware (determined by jumper settings). For example, to display 19.333 with one decimal point of precision by way of port 785 , one would type "DISPLAY $(19.333,1,785)$."

Internally, DISPLAY begins the process of translating the floating-point number into an appropriate sequence of output bytes by converting the number to a rightjustified string with the decimal point removed (lines 42-46). Next, the right-justified string, named $S$, is processed digit-by-digit by the FOR loop defined in line 48 .

The loop converts each character in the string to a byte that is then sent to the specified display circuitry by way of the port designated in variable $\mathbf{P}$. There are three possible conditions that may occur when building the output byte: (1) The LED will display a minus sign ("- "); (2) the LED will be blanked, and (3) the LED will display a number from 0 to 9 . In the first two cases, the threepart output byte, named BVL, is constructed by first calculating the address of the LED that will be manipulated. The address is calculated by simply subtracting 1 from the digit's position in $S$ (because digit addresses at 0 ) and adding the result directly to BVL (thereby placing the ad- dress in bits 0-2). Next, the EBCD code for " - " or " " " ( 10 or 15 , respectively) is placed in bits $3-6$ or BVL by shifting the EBCD code 3 bits to the left prior to their being added to BVL. Finally, 128 (mathematically equivalent to shifting 1 to the left by 7) is added to BVL to place a 1 in bit 7 so that the LED's decimal point is disabled.

The process for displaying a digit is similar, except that the EBCD value placed in bits 3-6 of BVL must be calculated (line 55) by subtracting 48 from the ASCII value of the digit. The process for assembling the three parts of the byte, however, are unchanged in all other respects, except that lines 57-58 enable the LED's decimal point by subtracting 128 from BVL (setting bit 7 to 0 ) if the LED coincides with the appropriate location for the decimal point (as specified by parameter D).

After outputting an "inverted" copy of BVL to the output port specified by parameter $P$, a small delay loop is executed in lines 63-63 to allow the LED hardware to "latch" the data. This process requires a minimum of $150 \mu \mathrm{sec}$. The loop in DISPLAY and Disp_off provides a $450-\mu \mathrm{sec}$ delay on a $20-\mathrm{MHz} 386$ machine and should be sufficient for any slower machines; significantly faster machines may require a larger loop. The assignment statement within the loop (i.e., $K:=J$ ) performs no function other than preventing optimizing compilers from removing what would otherwise constitute a null loop.

Procedure Disp_Off provides a convenient mechanism for blanking the entire display by setting each digit of the display to the EBCD code for " " "; the code for blanking the display is conceptually similar to the code for DISPLAY. 


\section{CONCLUSION}

The display-driver program and the LED display hardware illustrate the use of encoding/decoding address and data information with a single byte of data for controlling multiple outputs. Depending on the hardware that is used, the encoding/decoding scheme can use fewer or more bits for address/data information. For example, with a $4 \times 4$ encoding scheme ( 4 address bits and 4 data bits), one could control up to 64 different outputs from a single output port.

\section{REFERENCES}

IEE (1986). Hercules LED digital displays (pp. 41 -43). Van Nuys, CA. International Business Machines Corporation (1984). Personal computer hardware reference library-Serial/parallel adapter (1502494). Boca Raton, FL: IBM.

TAthaM, T. A., ZURN, K. R. (1989). The MED-PC experimental apparatus programming system. Behavior Research Methods. Instruments, \& Computers, 21, 294-302.

WYNNE, C. D. L. (1990). A Commodore 64-based interface system for the operant laboratory. Behavior Research Methods, Instruments, \& Computers, 22, 27-33.

\section{APPENDIX}

A Pascal Unit Containing Procedures DISPLAY and Disp_off, Which Simplify the Software aspects of Controlling the LED Display

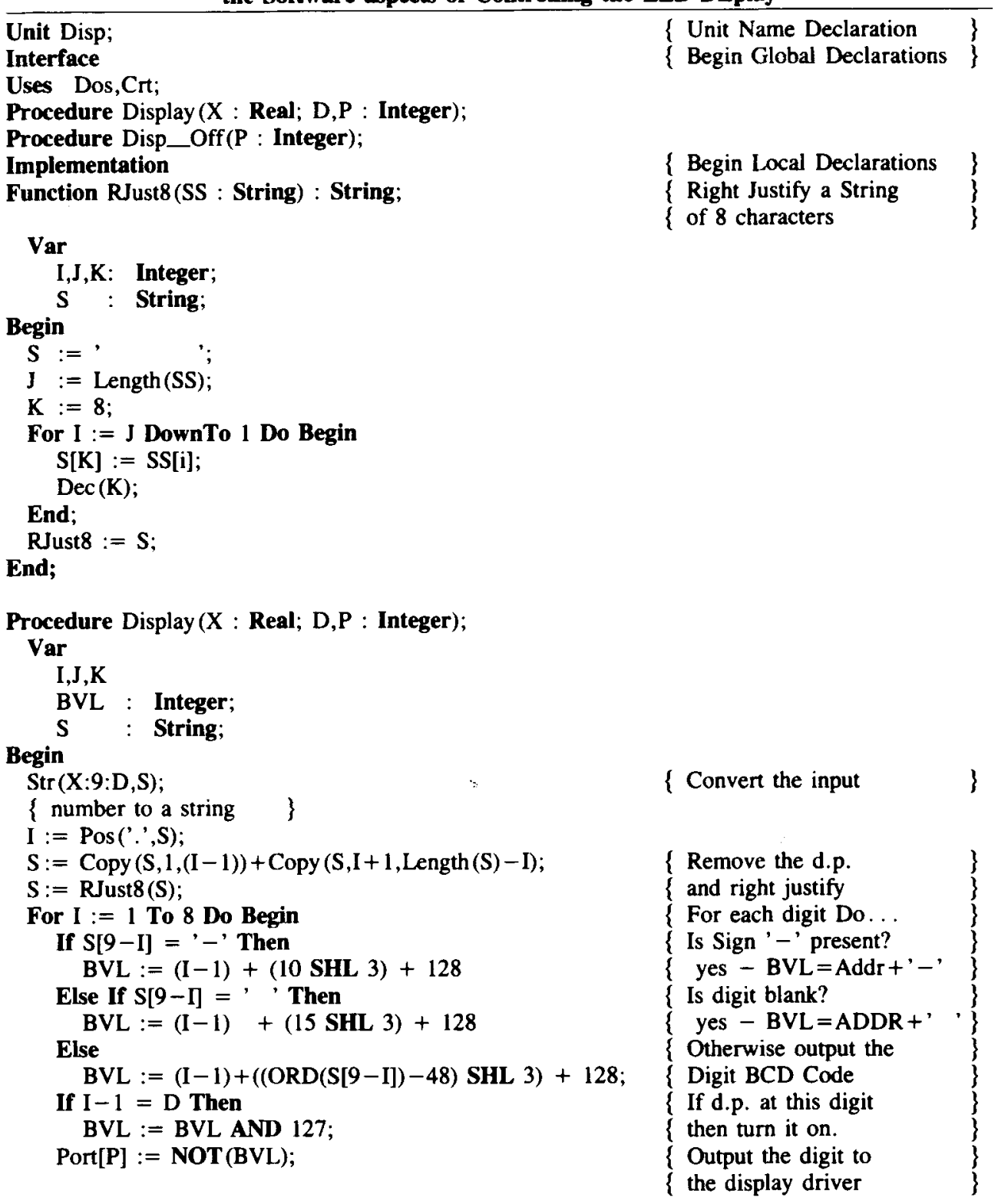


For $\mathrm{J}:=1$ To 250 Do \{ Require at least $150 \mu \mathrm{sec}$ delay for display hdwr $\mathbf{K}:=\mathbf{J}$

Approx $450 \mu \mathrm{sec}$ delay on $20-\mathrm{MHZ} 386$ might need adjustment for other machines...

End; \{ Digit Count Loop \}

End; \{ Procedure Display \}

Procedure Disp_Off(P : Integer);

Var

I,J,K,BVL : Integer;

Begin

For $\mathrm{I}:=1$ To 8 Do Begin

BVL := (I-1) + (15 SHL 3$)+128$

Port[P] := NOT $(B V L)$;

For $\mathrm{J}:=1$ To 250 Do

$\mathbf{K}:=\mathbf{J}$;

\{ Output' 'value to

$\{$ each digit address

$\{$ with d.p. off.

End;

$\{$ Delay Loop. .

End; \{ Procedure Disp_off \}

End. \{ Unit \} 